\title{
Spin-wave stiffness and micromagnetic exchange interactions expressed by means of the KKR Green function approach
}

\author{
S. Mankovsky, S. Polesya, and H. Ebert \\ Department of Chemistry/Phys. Chemistry, LMU Munich, Butenandtstrasse 11, D-81377 Munich, Germany
}

(Received 5 November 2018; revised manuscript received 8 March 2019; published 22 March 2019)

\begin{abstract}
We represent an approach to calculate micromagnetic model parameters such as the tensor of exchange stiffness, Dzyaloshinskii-Moriya interaction (DMI), as well as spin-wave stiffness. The scheme is based on the fully relativistic Korringa-Kohn-Rostoker Green function (KKR-GF) technique and can be seen as a relativistic extension of the work of Lichtenstein et al. [J. Magn. Magn. Mater. 67, 65 (1987)]. The expression for $D^{z \alpha}$ elements of DMI differ from the expressions for $D^{x \alpha}$ and $D^{y \alpha}$ elements as the former are derived via second-order perturbation term of the energy caused by spin-spiral while the latter are associated with the first-order term. Corresponding numerical results are compared with those obtained using other schemes reported in the literature.
\end{abstract}

DOI: 10.1103/PhysRevB.99.104427

\section{INTRODUCTION}

To map the DFT total energy onto the Heisenberg model and its extensions, different schemes have been reported in the literature [1-5], giving access to first-principles calculations of the exchange coupling parameters. An expression to calculate within multiple scattering theory (MST) or, equivalently, Korringa-Kohn-Rostoker Green function (KKR-GF) formalism the the isotropic exchange parameters entering the classical Heisenberg Hamiltonian has been derived first by Lichtenstein et al. [1]. The classical Heisenberg model has been extended to account for relativistic effects on the inter-atomic exchange interactions, accounting first of all for the Dzyaloshinskii-Moriya interaction (DMI). An approach to calculate the corresponding interaction parameters, also based on the MST formalism, was suggested by Udvardi et al. [4]. Both approaches mentioned above use the magnetic force theorem that allows to evaluate the energy change associated with a distortion of the magnetization of a system via the expression:

$$
\Delta \mathcal{E} \approx \int^{E_{F}} d E\left(E-E_{F}\right) \delta n(E)
$$

where $n(E)$ is the density of states (DOS) of the electrons. The use of multiple scattering formalism allows the direct calculation of the interatomic exchange interaction using Lloyd's formula [6], which gives the energy integrated DOS (NOS) $N(E)$. This leads to an explicit expression for the energy change due to the tilting of two magnetic moments in the FM ordered system and as a result to the exchange coupling parameters. Using the extended Heisenberg Hamiltonian $\mathcal{H}_{H}$ in the form suggested by Udvardi et al. [4],

$$
\mathcal{H}_{H}=-\frac{1}{2} \sum_{i \neq j} \mathbf{e}_{i} \underline{J}_{i j} \mathbf{e}_{j}+\sum_{i} K\left(\mathbf{e}_{i}\right),
$$

the isotropic exchange interaction and DMI parameters are deduced from the symmetric and antisymmetric parts of the exchange tensor $\underline{J}_{i j}$ :

$$
J_{i j}=\frac{1}{3} \operatorname{Tr} \underline{J}_{i j}
$$

and

$$
D_{i j}^{v}=\epsilon^{\lambda \mu \nu} \frac{J_{i j}^{\lambda \mu}-J_{i j}^{\mu \lambda}}{2}
$$

with $\epsilon^{\lambda \mu v}$ the Levi-Civita tensor. A similar formulation for the exchange tensor, also on the basis of MST, has been suggested in our previous work [5].

Adopting a micromagnetic approach the free-energy density may be expressed by [7]

$$
F(\vec{r})=\sum_{\alpha} A_{\alpha \alpha}\left(\frac{\partial \hat{m}}{\partial r_{\alpha}}\right)^{2}+\sum_{\alpha \nu} D^{\nu \alpha}\left(\hat{m} \times \frac{\partial \hat{m}}{\partial r_{\alpha}}\right)_{\nu} .
$$

Also in this case, the various parameters can be evaluated from first principles calculations. In particular, the spin-wave stiffness $\mathfrak{D}_{\alpha \alpha}$ can be evaluated from the second-order derivative of the spin-spiral energy $E(\vec{q})[8]$ :

$$
\mathfrak{D}_{\alpha \alpha}=\frac{2 g}{M} \frac{\partial^{2} E(\vec{q})}{\partial q_{\alpha} \partial q_{\alpha}} .
$$

The corresponding expression for the closely connected exchange stiffness $A_{\alpha \alpha}=\mathfrak{D}_{\alpha \alpha} M /(2 g)$ (where $g$ is the Landé factor and $M$ is the total magnetic moment [9]) has been derived by Liechtenstein et al. [10] by means of nonrelativistic multiple scattering theory.

Recently, Freimuth et al. [11] demonstrated that the parameters entering the relativistic free energy density in Eq. (3), i.e., the Dzyaloshinskii-Moriya interaction and exchange stiffness, can be computed by using the Berry phase approach. The microscopic DMI parameters in this case are evaluated as the slope of the spin-wave energy $E(\vec{q})$ at $\vec{q}=0$ :

$$
D^{\nu \alpha}=\left(\frac{\partial E\left\{[\hat{z} \times \delta \hat{m}(\vec{q})]_{\nu}\right\}}{\partial q_{\alpha}}\right)_{q=0} .
$$


In the present work we represent an approach for the calculation of the parameters of the Heisenberg and micromagnetic models performed on the same footing within the fully relativistic spin-polarized Korringa-Kohn-Rostoker Green function (KKR-GF) method. This approach, described in Sec. II B, is based on the expansion of the total energy in powers of wave vector components characterizing a spin spiral with a small $q$-values treated as a perturbation. Considering the ferromagnetic state as a reference state of the system, it is demonstrated in Sec. II C, that the spin-stiffness constant is associated with the second-order term of the energy expansion. Also, it will be shown in Sec. II D, that the expressions for the parameters $D^{x \alpha}$ and $D^{y \alpha}$ are given by the first-order term of the energy expansion, while $D^{z \alpha}$ is associated with the second-order energy term. In Sec. III we analyze the results of calculations of the spin-wave stiffness and the DMI tensor, which are based on the present approach, and compare these results with others calculations and with experimental data.

\section{THEORETICAL BACKGROUND BASED ON THE SPIN SPIRAL APPROACH}

\section{A. Representation of the electronic structure}

To derive explicit expressions for the various interaction parameters on the basis of electronic structure calculations, we start from the Dirac Hamiltonian set up within the framework of relativistic spin-density functional theory [12]:

$$
\mathcal{H}_{\mathrm{D}}=-i c \overrightarrow{\boldsymbol{\alpha}} \cdot \vec{\nabla}+\frac{1}{2} c^{2}(\boldsymbol{\beta}-1)+V(\vec{r})+\beta \overrightarrow{\boldsymbol{\sigma}} \cdot \vec{B}_{x c}(\vec{r}),
$$

where $\vec{B}_{x c}(\vec{r})$ is the spin-dependent part of the exchangecorrelation potential and all other quantities have there usual meaning $[13,14]$.

Instead of representing the electronic structure in terms of Bloch states derived from the Hamiltonian in Eq. (6) it is much more convienient for our purposes to use the electronic Green function $G\left(\vec{r}, \vec{r}^{\prime}, E\right)$ instead. Within the KKR-GF approach $G\left(\vec{r}, \vec{r}^{\prime}, E\right)$ is represented in real space by the expression [14]:

$$
\begin{aligned}
G\left(\vec{r}, \vec{r}^{\prime}, E\right)= & \sum_{\Lambda_{1} \Lambda_{2}} Z_{\Lambda_{1}}^{n}(\vec{r}, E) \tau_{\Lambda_{1} \Lambda_{2}}^{n n^{\prime}}(E) Z_{\Lambda_{2}}^{n^{\prime} \times}\left(\vec{r}^{\prime}, E\right) \\
& -\sum_{\Lambda_{1}}\left[Z_{\Lambda_{1}}^{n}(\vec{r}, E) J_{\Lambda_{1}}^{n \times}\left(\vec{r}^{\prime}, E\right) \Theta\left(r^{\prime}-r\right)\right. \\
& \left.\times J_{\Lambda_{1}}^{n}(\vec{r}, E) Z_{\Lambda_{1}}^{n \times}\left(\vec{r}^{\prime}, E\right) \Theta\left(r-r^{\prime}\right)\right] \delta_{n n^{\prime}} .
\end{aligned}
$$

Here $Z_{\Lambda_{1}}^{n}(\vec{r}, E)$ and $J_{\Lambda_{1}}^{n}(\vec{r}, E)$ are the regular and irregular solutions of the single site Dirac equation and $\underline{\tau}^{n n^{\prime}}$ is the so-called scattering path operator matrix [14].

The specific form of the Dirac Hamiltonian in Eq. (6) also allows to express the impact of the change in the potential $\Delta V(\vec{r})$ due to the rotation of the magnetic moments on the atomic sites in a very simple way. Assuming that $\vec{B}_{x c}(\vec{r})$ on site $i$ is aligned along the orientation of the spin moment $\hat{m}_{i}$, i.e., $\vec{B}_{x c}(\vec{r})=B_{x c}(\vec{r}) \hat{m}_{i}$, and taking into account that $\hat{m}_{i}=\hat{z}$ for a ferromagnetic (FM) state, the potential change $\Delta V(\vec{r})$ connected with the tilting of rigid magnetic moments has the form

$$
\Delta V(\vec{r})=\sum_{i} \beta\left(\vec{\sigma} \cdot \hat{m}_{i}-\sigma_{z}\right) B_{x c}(\vec{r})
$$

\section{B. Basic properties of the exchange interactions}

Similar to our previous work [5], the present approach is based on the magnetic force theorem. As a starting point we use the ferromagnetic (FM) state as a reference state and neglect for the moment all temperature effects, i.e., assume $T=0 \mathrm{~K}$. In this case, a change of the total energyg caused by the formation of a spin spiral in the system is given by Eq. (1). However, instead of using the Lloyd formula, we represent the change of the density of states $\Delta n(E)$ in terms of the Green function $G_{0}(E)$ for the FM reference state, which is modified due to the perturbation. Denoting the corresponding change in the Green function $\Delta G(E)$ and neglecting temperature effects one can write the change of the total energy:

$$
\Delta \mathcal{E} \approx-\frac{1}{\pi} \operatorname{Im} \operatorname{Tr} \int^{E_{F}} d E\left(E-E_{F}\right) \Delta G(E),
$$

where $E_{F}$ is the Fermi energy. Assuming that the perturbation is small, the induced change of the Green function can be represented by the following perturbation expansion

$$
\begin{aligned}
\Delta G(E)= & G_{0}(E) \Delta V G_{0}(E) \\
& +G_{0}(E) \Delta V G_{0}(E) \Delta V G_{0}(E)+\ldots,
\end{aligned}
$$

where $\Delta V$ is a perturbation operator, Eq. (8), describing the creation of a spin-spiral in the FM system. Substituting Eq. (10) into Eq. (9) and using the sum rule $\frac{d G}{d E}=-G G$ for the Green function, one obtains an expression for the total energy change associated with the spin spiral:

$$
\begin{aligned}
\Delta \mathcal{E}= & -\frac{1}{\pi} \operatorname{Im} \operatorname{Tr} \int^{E_{F}} d E\left(E-E_{F}\right) G_{0}(E) \Delta V G_{0}(E) \\
& +\frac{1}{\pi} \operatorname{Im} \operatorname{Tr} \int^{E_{F}} d E\left(E-E_{F}\right) \Delta V G_{0}(E) \Delta V \frac{d G_{0}(E)}{d E} .
\end{aligned}
$$

By performing integration by parts for the second equation in Eq. (11) and taking into account that $(E-$ $\left.E_{F}\right)\left.\Delta V G_{0}(E) \Delta V G_{0}(E)\right|_{E=E_{F}}=0$, the total energy change $\Delta \mathcal{E}$ is given by

$$
\begin{aligned}
\Delta \mathcal{E}= & -\frac{1}{\pi} \operatorname{Im} \operatorname{Tr} \int^{E_{F}} d E\left(E-E_{F}\right) G_{0}(E) \Delta V G_{0}(E) \\
& -\frac{1}{\pi} \operatorname{Im} \operatorname{Tr} \int^{E_{F}} d E \Delta V G_{0}(E) \Delta V G_{0}(E) \\
= & K^{(1)}+K^{(2)}
\end{aligned}
$$

Here only the first- and second-order terms of the expansion are kept as they are responsible for the effects discussed below. Note, however, that higher-order terms can also be nonnegligible leading to corresponding higher-order exchange interaction terms in the Heisenberg Hamiltonian, which, however, are not discussed in the present work.

\section{Spin-wave stiffness}

We first consider a spin spiral characterized by its wave vector $\vec{q}$, with the magnetic moment direction on site $(i)$ given 
by the expression

$$
\hat{m}_{i}=\left(\sin \theta \cos \left(\vec{q} \cdot \vec{R}_{i}\right), \sin \theta \sin \left(\vec{q} \cdot \vec{R}_{i}\right), \cos \theta\right),
$$

implying the same cone angle $\theta$ for all atomic sites.
For the sake of convenience, we start with the Heisenberg model. When the spin-spiral is given in the form of Eq. (14), one gets the following change in energy $\Delta E_{H}$ with respect to the FM state with its magnetization direction along $\hat{z}$,

$$
\begin{aligned}
\Delta E_{H}= & -\theta \sum_{i \neq j}^{N} D_{i j}^{x}\left[\sin \left(\vec{q} \cdot \vec{R}_{i}\right)-\sin \left(\vec{q} \cdot \vec{R}_{j}\right)\right]-\theta^{2} \sum_{i \neq j}^{N}\left\{D_{i j}^{z} \sin \left(\vec{q} \cdot \vec{R}_{j}-\vec{R}_{i}\right)+\frac{1}{2}\left[\left(J_{i j}^{x y}+J_{i j}^{y x}\right) \sin \left(\vec{q} \cdot \vec{R}_{j}+\vec{R}_{i}\right)\right.\right. \\
& \left.\left.+\left(J_{i j}^{x x}-J_{i j}^{y y}\right) \cos \left(\vec{q} \cdot \vec{R}_{j}+\vec{R}_{i}\right)+\left(J_{i j}^{x x}+J_{i j}^{y y}\right) \cos \left(\vec{q} \cdot \vec{R}_{j}-\vec{R}_{i}\right)\right]\right\}+\ldots
\end{aligned}
$$

where we restrict the expansion up to second order with respect to the angle $\theta$, and focus in the following on the term proportional to $\theta^{2}$.

Using the expressions for the Green function given in Eq. (7) and for the perturbation due to the formation of a spin spiral according to Eq. (8), one obtains an expression for the free energy contribution $K^{(2)}$ given in the multiple scattering representation

$$
\begin{aligned}
K^{(2)}= & -\frac{\theta^{2}}{\pi} \sum_{i \neq j}^{N} \operatorname{Im} \operatorname{Tr} \int^{E_{F}} d E\left\{\frac{1}{2}\left(\underline{T}_{x}^{i} \underline{\tau}^{i j} \underline{T}_{x}^{j} \underline{\tau}^{j i}+\underline{T}_{y}^{i} \underline{\tau}^{i j} \underline{T}_{y}^{j} \underline{\tau}^{j i}\right) \cos \left[\vec{q} \cdot\left(\vec{R}_{j}-\vec{R}_{i}\right)\right]\right. \\
& +\frac{1}{2}\left(\underline{T}_{x}^{i} \underline{\tau}^{i j} \underline{T}_{x}^{j} \underline{\tau}^{j i}-\underline{T}_{y}^{i} \underline{\tau}^{i j} \underline{T}_{y}^{j} \underline{\tau}^{j i}\right) \cos \left[\vec{q} \cdot\left(\vec{R}_{j}+\vec{R}_{i}\right)\right] \\
& +\frac{1}{2}\left(\underline{T}_{x}^{i} \underline{\tau}^{i j} \underline{T}_{y}^{j} \underline{\tau}^{j i}-\underline{T}_{y}^{i} \underline{\tau}^{i j} \underline{T}_{x}^{j} \underline{\tau}^{j i}\right) \sin \left[\vec{q} \cdot\left(\vec{R}_{j}-\vec{R}_{i}\right)\right] \\
& \left.+\frac{1}{2}\left(\underline{T}_{x}^{i} \underline{\tau}^{i j} \underline{T}_{y}^{j} \underline{\tau}^{j i}+\underline{T}_{y}^{i} \underline{\tau}^{i j} \underline{T}_{x}^{j} \underline{\tau}^{j i}\right) \sin \left[\vec{q} \cdot\left(\vec{R}_{j}+\vec{R}_{i}\right)\right]\right\} .
\end{aligned}
$$

Considering for the sake of simplicity a system with one atom per unit cell one has the following matrix elements representing the change in the potential

$$
\begin{aligned}
& T_{x, \Lambda_{1} \Lambda_{2}}(E)=\int_{\Omega_{0}} d^{3} r Z_{\Lambda_{1}}^{\times}(\vec{r}, E) \beta \sigma_{x} B_{x c}(\vec{r}) Z_{\Lambda_{2}}(\vec{r}, E), \\
& T_{y, \Lambda_{1} \Lambda_{2}}(E)=\int_{\Omega_{0}} d^{3} r Z_{\Lambda_{1}}^{\times}(\vec{r}, E) \beta \sigma_{y} B_{x c}(\vec{r}) Z_{\Lambda_{2}}(\vec{r}, E),
\end{aligned}
$$

with $\underline{T}_{\mu}^{i}=\underline{T}_{\mu}^{j}=\underline{T}_{\mu}$. By doing a one-to-one comparison of the energy terms associated with a pair of sites $(i, j)$ as given by Eqs. (15) and (16), respectively, one gets expressions for the elements $J_{i j}^{y x}$ and $J_{i j}^{x y}$ of the $\underline{J}_{i j}$ tensor, as well as the interatomic DMI terms $D_{i j}^{z}$, which have the same form as those derived previously by us using the Lloyd formula [5].

In contrast to our previous work, the goal of the present study is to get expressions for the micromagnetic interaction parameters. This is achieved by performing a Fourier transformation for the scattering path operator

$$
\underline{\tau}^{i j}=\frac{1}{\Omega_{B Z}} \int_{\Omega_{B Z}} d^{3} k e^{i \vec{k}\left(R_{j}-R_{i}\right)} \underline{\tau}(\vec{k})
$$

leading to an alternative expression for the energy change caused by the formation of a spin-spiral

$$
\begin{aligned}
K^{(2)}= & -\frac{\theta^{2}}{2 \pi} \operatorname{Im} \operatorname{Tr} \int^{E_{F}} d E \frac{1}{\Omega_{B Z}} \int_{\Omega_{B Z}} d^{3} k \frac{1}{\Omega_{B Z}} \int_{\Omega_{B Z}} d^{3} k^{\prime} \\
& \times\left\{\frac{1}{2}\left[\underline{T}_{x} \underline{\tau}(\vec{k}, E) \underline{T}_{x} \underline{\tau}\left(\vec{k}^{\prime}, E\right)+\underline{T}_{y} \underline{\tau}(\vec{k}, E) \underline{T}_{y} \underline{\tau}\left(\vec{k}^{\prime}, E\right)\right]\left[\delta\left(\vec{k}+\vec{q}-\vec{k}^{\prime}\right)+\delta\left(\vec{k}-\vec{q}-\vec{k}^{\prime}\right)\right]\right. \\
& +\frac{1}{2 i}\left[\underline{T}_{x} \underline{\tau}(\vec{k}, E) \underline{T}_{y} \underline{\tau}\left(\vec{k}^{\prime}, E\right)-\underline{T}_{y} \underline{\tau}(\vec{k}, E) \underline{T}_{x} \underline{\tau}\left(\vec{k}^{\prime}, E\right)\right]\left[\delta\left(\vec{k}+\vec{q}-\vec{k}^{\prime}\right)-\delta\left(\vec{k}-\vec{q}-\vec{k}^{\prime}\right)\right] \\
& +\frac{1}{2}\left[\underline{T}_{x} \underline{\tau}(\vec{k}, E) \underline{T}_{x} \underline{\tau}\left(\vec{k}^{\prime}, E\right)-\underline{T}_{y} \underline{\tau}(\vec{k}, E) \underline{T}_{y} \underline{\tau}\left(\vec{k}^{\prime}, E\right)\right]\left[\delta\left(\vec{k}+\vec{q}-\vec{k}^{\prime}\right)+\delta\left(\vec{k}-\vec{q}-\vec{k}^{\prime}\right)\right] \delta\left(\vec{k}-\vec{k}^{\prime}\right) \\
& \left.+\frac{1}{2 i}\left[\underline{T}_{x} \underline{\tau}(\vec{k}, E) \underline{T}_{y} \underline{\tau}\left(\vec{k}^{\prime}, E\right)+\underline{T}_{y} \underline{\tau}(\vec{k}, E) \underline{T}_{x} \underline{\tau}\left(\vec{k}^{\prime}, E\right)\right]\left[\delta\left(\vec{k}+\vec{q}-\vec{k}^{\prime}\right)-\delta\left(\vec{k}-\vec{q}-\vec{k}^{\prime}\right)\right] \delta\left(\vec{k}-\vec{k}^{\prime}\right)\right\} .
\end{aligned}
$$


The last term in Eq. (19) is equal to zero, while the third one corresponds to the nonlocal MCA discussed by Udvardi et al. [4], which gives for the present geometry the contribution to the MCA within the $x y$ plane

$$
\Delta E_{\mathrm{MCA}}=-\frac{\theta^{2}}{2 \pi} \operatorname{Im} \operatorname{Tr} \int^{E_{F}} d E \frac{1}{\Omega_{B Z}} \int_{\Omega_{B Z}} d^{3} k\left[\underline{T}_{x} \underline{\tau}(\vec{k}, E) \underline{T}_{x} \underline{\tau}(\vec{k}, E)-\underline{T}_{y} \underline{\tau}(\vec{k}, E) \underline{T}_{y} \underline{\tau}(\vec{k}, E)\right] .
$$

To consider the first two terms in Eq. (19), one can use a Taylor expansion of the $\tau$ matrix for small $\vec{q}$ vectors,

$$
\underline{\tau}(\vec{k} \pm \vec{q}, E)=\underline{\tau}(\vec{k}, E) \pm \sum_{\alpha} \frac{\partial \underline{\tau}(\vec{k}, E)}{\partial k_{\alpha}} q_{\alpha}+\frac{1}{2} \sum_{\alpha, \beta} \frac{\partial^{2} \underline{\tau}(\vec{k}, E)}{\partial k_{\alpha} \partial k_{\beta}} q_{\alpha} q_{\beta},
$$

which gives the corresponding contribution $K_{1-2}^{(2)}$ to $K^{(2)}$

$$
\begin{aligned}
K_{1-2}^{(2)}= & -\frac{\theta^{2}}{2 \pi} \operatorname{Im} \operatorname{Tr} \int^{E_{F}} d E \frac{1}{\Omega_{B Z}} \int_{\Omega_{B Z}} d^{3} k \frac{1}{2}\left[\underline{T}_{x} \underline{\tau}(\vec{k}, E) \underline{T}_{x}(2 \underline{\tau}(\vec{k}, E)+\underbrace{\left.\sum_{\alpha, \beta} \frac{\partial^{2} \underline{\tau}(\vec{k}, E)}{\partial k_{\alpha} \partial k_{\beta}} q_{\alpha} q_{\beta}\right)}_{T 1}\right. \\
& +\underline{T}_{y} \underline{\tau}(\vec{k}, E) \underline{T}_{y}(2 \underline{\tau}(\vec{k}, E)+\underbrace{\left.\sum_{\alpha, \beta} \frac{\partial^{2} \underline{\tau}(\vec{k}, E)}{\partial k_{\alpha} \partial k_{\beta}} q_{\alpha} q_{\beta}\right)}_{T 2} \\
& \left.+\frac{1}{i}\left(\underline{T}_{x} \underline{\tau}(\vec{k}, E) \underline{T}_{y} \sum_{\alpha} 2 \frac{\partial \underline{\tau}(\vec{k}, E)}{\partial k_{\alpha}} q_{\alpha}-\underline{T}_{y} \underline{\tau}(\vec{k}, E) \underline{T}_{x} \sum_{\alpha} 2 \frac{\partial \underline{\tau}(\vec{k}, E)}{\partial k_{\alpha}} q_{\alpha}\right)\right] .
\end{aligned}
$$

Doing an integration by parts for the expression involving the term $T 1$ indicated in Eq. (22) (see Ref. [15]), one obtains

$$
\begin{aligned}
T 1 & =-\frac{\theta^{2}}{2 \pi} \operatorname{Im} \operatorname{Tr} \int^{E_{F}} d E \frac{1}{\Omega_{B Z}} \int_{\Omega_{B Z}} d^{3} k \frac{1}{2} \underline{T}_{x} \underline{\tau}(\vec{k}, E) \underline{T}_{x} \sum_{\alpha, \beta} \frac{\partial^{2} \underline{\tau}(\vec{k}, E)}{\partial k_{\alpha} \partial k_{\beta}} q_{\alpha} q_{\beta} \\
& =\frac{\theta^{2}}{4 \pi} \sum_{\alpha, \beta} q_{\alpha} q_{\beta} \operatorname{Im} \operatorname{Tr} \int^{E_{F}} d E \frac{1}{\Omega_{B Z}} \int_{\Omega_{B Z}} d^{3} k \underline{T}_{x} \frac{\partial \underline{\tau}(\vec{k}, E)}{\partial k_{\alpha}} \underline{T}_{x} \frac{\partial \underline{\tau}(\vec{k}, E)}{\partial k_{\beta}} .
\end{aligned}
$$

The same transformation can also be made for the term $T 2$.

Equating now the second order derivatives with respect to the $\vec{q}$ vector for the microscopic and model energies in the limit $q \rightarrow 0$,

$$
\left(\frac{\partial^{2} \Delta \mathcal{E}}{\partial q_{\alpha} \partial q_{\beta}}\right)_{q=0}=\left(\frac{\partial^{2} K^{(2)}}{\partial q_{\alpha} \partial q_{\beta}}\right)_{q=0}=\left(\frac{\partial^{2} \Delta E_{H}}{\partial q_{\alpha} \partial q_{\beta}}\right)_{q=0},
$$

one obtains the components of the exchange tensor. This leads to an expression for the spin-wave stiffness

$$
\mathfrak{D}_{\alpha \beta}=\frac{1}{\theta^{2}} \frac{4}{M} \frac{\partial^{2} E}{\partial q_{\alpha} \partial q_{\beta}}=\frac{1}{\pi M} \operatorname{Im} \operatorname{Tr} \int^{E_{F}} d E \frac{1}{\Omega_{B Z}} \int_{\Omega_{B Z}} d^{3} k\left[\underline{T}_{x} \frac{\partial \underline{\tau}(\vec{k}, E)}{\partial k_{\alpha}} \underline{T}_{x} \frac{\partial \underline{\tau}(\vec{k}, E)}{\partial k_{\beta}}+\underline{T}_{y} \frac{\partial \underline{\tau}(\vec{k}, E)}{\partial k_{\alpha}} \underline{T}_{y} \frac{\partial \underline{\tau}(\vec{k}, E)}{\partial k_{\beta}}\right],
$$

which can be seen as a relativistic generalization of the expression given by Liechtenstein et al. [1].

\section{Dzyaloshinskii-Moriya interaction}

Taking the first-order derivative of $K_{1-2}^{(2)}$ with respect to the components of the $\vec{q}$-vector the last term in Eq. (19) gives in the limit $q \rightarrow 0$ the elements $D^{z \alpha}$ of the DMI tensor:

$$
D^{z \alpha}=\frac{1}{\theta^{2}} \lim _{q \rightarrow 0} \frac{\partial K^{(2)}}{\partial q_{\alpha}}=\left(\frac{1}{2 \pi}\right) \operatorname{Re} \operatorname{Tr} \int^{E_{F}} d E \frac{1}{\Omega_{B Z}} \int_{\Omega_{B Z}} d^{3} k\left[\underline{T}_{x} \underline{\tau}(\vec{k}, E) \underline{T}_{y} \frac{\partial \underline{\tau}(\vec{k}, E)}{\partial k_{\alpha}}-\underline{T}_{y} \underline{\tau}(\vec{k}, E) \underline{T}_{x} \frac{\partial \underline{\tau}(\vec{k}, E)}{\partial k_{\alpha}}\right] .
$$

To calculate the tensor elements $D^{x \alpha}$ and $D^{y \alpha}$, it is convenient to use a spin spiral given in the following rather general form [16]:

$$
\hat{m}_{i}=\hat{m}_{\mu} \sin \left(\vec{q} \cdot \vec{R}_{i}\right)+\hat{m}_{z} \cos \left(\vec{q} \cdot \vec{R}_{i}\right)
$$


where $\hat{m}_{i}$ characterizes the direction of the magnetic moments on site $R_{i}$ with $\hat{m}_{i} \equiv \hat{m}\left(\vec{R}_{i}\right), \mu=\{x, y\}$ and the wave vector $\vec{q}$ can have any direction. Assuming a weak deviation of the magnetic moments $\vec{m}_{i}$ from the $\hat{z}$ direction, this setting allows to get rid of the first-order derivatives with respect to $q_{\alpha}$, related to the term $K^{(2)}$ in Eq. (13) and to focus on the term $K^{(1)}$.

With this, the elements $D^{\mu \alpha}$ of the micromagnetic tensor representing the DMI as defined by Eq. (5) are determined exclusively by the first-order term $K^{(1)}$ in Eq. (13). The term $K^{(1)}$ associated with the perturbation Eq. (8) induced by a spin spiral as described by Eq. (27) has the following form:

$$
\begin{aligned}
K^{(1)}= & -\frac{1}{2 \pi} \sum_{i \neq j} \operatorname{Im} \operatorname{Tr} \int^{E_{F}} d E\left(E-E_{F}\right) \\
& \times\left\{\sin \left[\vec{q} \cdot\left(\vec{R}_{i}-\vec{R}_{j}\right)\right][\underbrace{\underline{O}^{j}(E) \underline{\tau}^{j i}(E) \underline{T}_{\mu}^{i}(E) \underline{\tau}^{i j}(E)}_{T 1}-\underbrace{\underline{T}_{\mu}^{j}(E) \underline{\tau}^{j i}(E) \underline{O}^{i}(E) \underline{\tau}^{i j}(E)}_{T 2}]\right. \\
& \left.+\left[\cos \left[\vec{q} \cdot\left(\vec{R}_{i}-\vec{R}_{j}\right)\right]-1\right][\underbrace{\underline{O}^{j}(E) \underline{\tau}^{j i}(E) \underline{T}_{z}^{i}(E) \underline{\tau}^{i j}(E)}_{T 3}-\underbrace{\underline{T}_{z}^{j}(E) \underline{\tau}^{j i}(E) \underline{O}^{i}(E) \underline{\tau}^{i j}(E)}_{T 4}]\right\} .
\end{aligned}
$$

In the case of one atom per unit cell one has for the matrices occurring in Eq. (28) $\underline{O}^{i}(E)=\underline{O}(E)$ and $\underline{T}_{\mu}^{i}(E)=\underline{T}_{\mu}(E)$, with $\underline{T}_{\mu}(E)$ given by Eq. (17) and the overlap matrix given by

$$
O_{\Lambda_{1} \Lambda_{2}}(E)=\int_{\Omega_{0}} d^{3} r Z_{\Lambda_{1}}^{\times}(\vec{r}, E) Z_{\Lambda_{2}}(\vec{r}, E)
$$

Calculating the derivative $\frac{\partial K^{(1)}}{\partial q_{y}}$ in the limit $q \rightarrow 0$, the terms $T 1$ and $T 2$ in Eq. (28) giving the only nonvanishing contributions to the DMI parameters are

$$
\begin{aligned}
T 1 \rightarrow & -\frac{1}{\pi} \lim _{q \rightarrow 0} \frac{\partial}{\partial q_{\alpha}}\left\{\operatorname{Im} \operatorname{Tr} \frac{1}{2 i} \int^{E_{F}} d E\left(E-E_{F}\right)\right. \\
& \left.\times\left[\underline{O}(E) \frac{1}{\Omega_{B Z}} \int_{\Omega_{B Z}} d^{3} k \underline{\tau}(\vec{k}, E) \underline{T}_{\mu}(E) \underline{\tau}(\vec{k}-\vec{q}, E)-\underline{O}(E) \frac{1}{\Omega_{B Z}} \int_{\Omega_{B Z}} d^{3} k \underline{\tau}(\vec{k}, E) \underline{T}_{\mu}(E) \underline{\tau}(\vec{k}+\vec{q}, E)\right]\right\} \\
T 2 \rightarrow & -\frac{1}{\pi} \lim _{q \rightarrow 0} \frac{\partial}{\partial q_{\alpha}}\left\{\operatorname{Im} \operatorname{Tr} \frac{1}{2 i} \int^{E_{F}} d E\left(E-E_{F}\right)\right. \\
& \left.\times\left[\underline{T}_{\mu}(E) \frac{1}{\Omega_{B Z}} \int_{\Omega_{B Z}} d^{3} k \underline{\tau}(\vec{k}, E) \underline{O}(E) \underline{\tau}(\vec{k}-\vec{q}, E)-\underline{T}_{\mu}(E) \frac{1}{\Omega_{B Z}} \int_{\Omega_{B Z}} d^{3} k \underline{\tau}(\vec{k}, E) \underline{O}(E) \underline{\tau}(\vec{k}+\vec{q}, E)\right]\right\} .
\end{aligned}
$$

Equating for the microscopic and model energies the derivatives with respect to components of the $\vec{q}$-vector one obtains in the limit $\vec{q} \rightarrow 0$ the elements $D^{\mu \alpha}$ of the micromagnetic DMI tensor:

$$
\begin{aligned}
D^{\mu \alpha}= & \lim _{q \rightarrow 0} \frac{\partial}{\partial q_{\alpha}} K^{(1)}=\epsilon_{\mu \nu} \frac{1}{\pi} \operatorname{Re} \operatorname{Tr} \int^{E_{F}} d E\left(E-E_{F}\right) \\
& \times \frac{1}{\Omega_{B Z}} \int d^{3} k\left[\underline{O}(E) \underline{\tau}(\vec{k}, E) \underline{T}_{\nu}(E) \frac{\partial}{\partial k_{\alpha}} \underline{\tau}(\vec{k}, E)-\underline{T}_{\nu}(E) \underline{\tau}(\vec{k}, E) \underline{O}(E) \frac{\partial}{\partial k_{\alpha}} \underline{\tau}(\vec{k}, E)\right],
\end{aligned}
$$

with $\mu=\{x, y\}$ and $\nu=\{x, y\}$ and $\epsilon_{\mu \nu}$ the elements of the transverse Levi-Civita tensor $\underline{\epsilon}=\left[\begin{array}{cc}0 & 1 \\ -1 & 0\end{array}\right]$.

This formulation obviously gives access to a discussion of the DMI parameters in terms of specific features of the electronic band structure in a similar way as suggested in Ref. [17]. As the present formulation is given within the KKR-GF formalism, it allows to deal both with ordered and disordered materials, where disorder may be treated using the coherent potential approximation (CPA) alloy theory.

In addition, it is worth noting that only the elements $D^{x \alpha}$ and $D^{y \alpha}$ are defined for the FM state with its magnetization along $\hat{z}$ direction, as only the $x$ and $y$ directions are allowed for a change of the transverse spin moment component. The elements $D^{x \alpha}$ and $D^{y \alpha}$ originate from the interatomic DMI components $D_{i j}^{x}$ and $D_{i j}^{y}$, respectively, characterizing for $\hat{m} \| \hat{z}$ the nonzero magnetic torques acting on one atom $i(j)$ from another atom at site $j(i)$ (see, e.g., Ref. [18]). As it follows from Eq. (15), the terms related to $D_{i j}^{x}$ and $D_{i j}^{y}$ appear in first-order within an expansion of the energy with respect to the angle $\theta$ characterizing the deviation of a magnetic moment from the $\hat{z}$ direction. However, the DMI component $D_{i j}^{z}$ and analogously the element $D^{z z}$ of the DMI tensor give the contribution to the energy which is of the order of $\theta^{2}$ : Since the DMI component $D_{i j}^{z}$ couples the components of the magnetic moments of atoms $i$ and $j, m_{i, j}^{x} \sim \theta$ and $m_{i, j}^{y} \sim \theta$, respectively, both components should be nonzero to give a contribution to the energy change; in contrast to the terms $D_{i j}^{x(y)}$ that couple the components $m^{z}$ and $m_{i, j}^{x(y)} \sim \theta$ of the magnetic moments. 


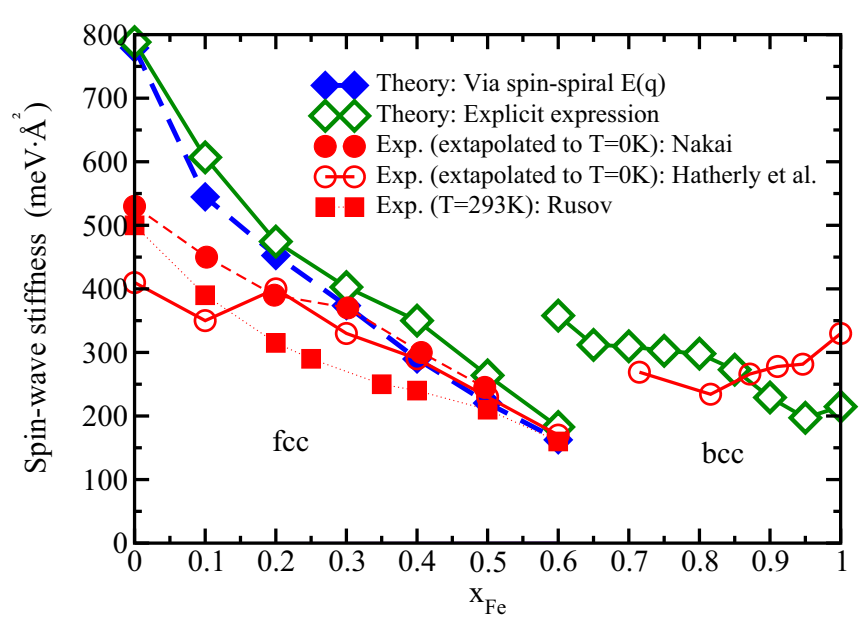

FIG. 1. Calculated spin-wave stiffness $\mathfrak{D}_{x x}$ (see text) of $\mathrm{Fe}_{1-x} \mathrm{Ni}_{x}$ alloys, with fcc (left) and bcc (right) crystal structures, represented as a function of the concentration in comparison with experiment: Nakai [19] (filled circles), Hatherly et al. [20] (open circles), and Rusov [21] (filled squares).

\section{RESULTS}

Figure 1 represents the spin-wave stiffness parameter $\mathfrak{D}_{x x}$ for the body-centered cubic (bcc) and face-centered cubic (fcc) phases of disordered $\mathrm{Fe}_{1-x} \mathrm{Ni}_{x}$ alloys calculated using Eq. (25) (open diamonds).

In the case of the fcc alloys, the results are compared with the spin-wave stiffness deduced from the energy dispersion $E(\vec{q})$ of a spin-spiral described by means of the generalized Bloch theorem and neglecting spin-orbit coupling (SOC) [22] (full diamonds). In spite of the very different approaches used, both curves are close to each other over the whole range of concentration considered. However, one should stress that a very dense $\mathrm{k}$-mesh is required to obtain reliable results for the Brillouin zone (BZ) integral in Eq. (25). For that reason a sequence of calculations with an increasing number of $\mathrm{k}$ points has been performed to ensure convergence. The final results have been obtained using a $144 \times 144 \times 144$ k-mesh spanning the whole BZ.

Figure 1 shows in addition experimental data obtained using different techniques. As one can see, the calculations reproduce the experimental data fairly well. For the bcc as well as fcc alloys, agreement between theory and experiment is best in the regime of concentrated alloys and gets less satisfying when approaching the $\mathrm{Fe}$ or $\mathrm{Ni}$, respectively, rich regimes.

To demonstrate the application of the derived expression for the micromagnetic DMI tensor, we consider here two different noncentrosymmetric system types, $\mathrm{Mn}_{1-x} \mathrm{Fe}_{x} \mathrm{Ge}$ and $\mathrm{Co}_{1-x} \mathrm{Fe}_{x} \mathrm{Ge}$, having the cubic B20 structure, and the strongly anisotropic multilayer system $\left(\mathrm{Cu} / \mathrm{Fe}_{1-x} \mathrm{Co}_{x} / \mathrm{Pt}\right)_{n}$. Focusing first on the B20 systems, Fig. 2 represents results for the three diagonal elements $D^{x x}, D^{y y}$, and $D^{z z}$ of the micromagnetic DMI tensor, with the $D^{x x}$ and $D^{y y}$ terms calculated in two different ways.

Assuming first the magnetization to be oriented along $\hat{z}$, the elements $D^{x x}$ and $D^{y y}$ (dotted lines and full squares, respectively, in Fig. 2) were calculated on the basis of Eq. (32) by integrating over the Brillouin zone. In this case, convergence

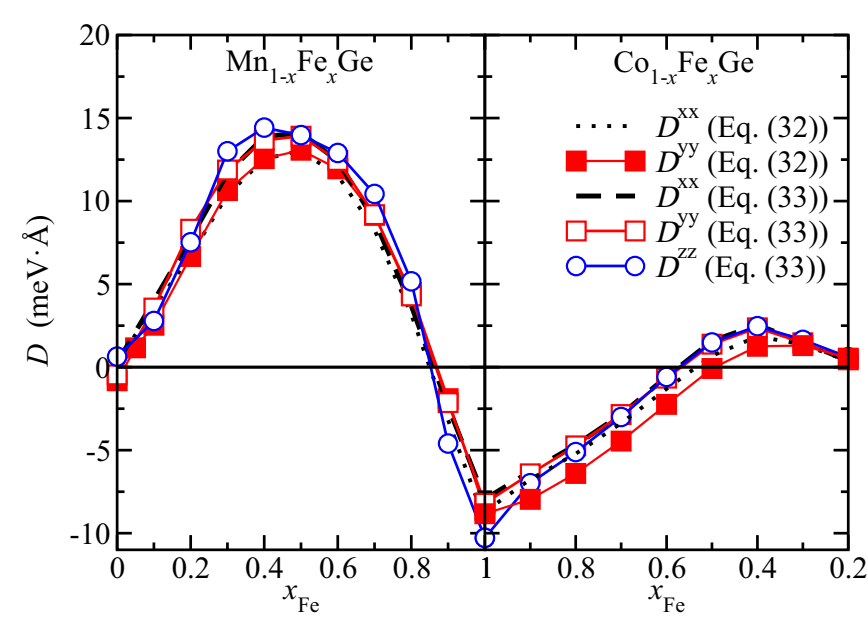

FIG. 2. The elements $D^{x x}, D^{y y}$, and $D^{z z}$ of the micromagnetic DMI tensor calculated for $\mathrm{Mn}_{1-x} \mathrm{Fe}_{x} \mathrm{Ge}$ (left) and $\mathrm{Co}_{1-x} \mathrm{Fe}_{x} \mathrm{Ge}$ represented as a function of $\mathrm{Fe}$ concentration. The results for $D^{x x}$ and $D^{y y}$ based on Eq. (33) were obtained calculating $D_{i j}^{x}$ and $D_{i j}^{y}$ as described in Ref. [16]. The corresponding result for $D^{z z}$, however, is based on $D_{i j}^{z}$ calculated as described in Ref. [5].

with respect to the number of $k$-points is faster when compared to the spin-wave stiffness calculations, and the results presented in Fig. 2 could be obtained using a $60 \times 60 \times 60$ $k$-mesh covering the whole BZ.

The second set of results for $D^{x x}$ and $D^{y y}$ shown in Fig. 2 (dashed line and open squares, respectively) is calculated using the expression

$$
D^{\alpha \alpha}=\sum_{j \neq 0} D_{0 j}^{\alpha}\left(\vec{R}_{j}-\vec{R}_{0}\right)_{\alpha},
$$

which is based on the interatomic DMI elements $D_{i j}^{x}$ and $D_{i j}^{y}$ [16]. To ensure convergence of the real-space summation in this expression all atomic sites $j$ with $\left|\vec{R}_{j}-\vec{R}_{0}\right| \leqslant 6.5 a$ and occupied by the transition metals have been included, where $a$ is the lattice parameter. The very small difference between the two sets of results can be attributed first of all to this restricted summation and excluding the indirect influence of the Ge atoms.

Concerning the calculation of the $D^{z z}$ element using the expression in Eq. (26), one has to point out that it has a much slower convergence with respect to the number of $k$-points when compared to calculations of $D^{x x}$ and $D^{y y}$. Although the structure of the integrand in Eq. (26) is very similar concerning the k-dependent scattering path operators when compared with Eq. (32) for $D^{x x}$ and $D^{y y}$, the corresponding BZ integrals behave very different because of the different weighting factors. This makes the calculation of $D^{z z}$ via Eq. (26) much more demanding when compared to calculations of $D^{x x}$ and $D^{y y}$. Therefore, $D^{z z}$ has been calculated here only using Eq. (33) (open circles in Fig. 2), with $D_{i j}^{z}$ obtained via an expression reported previously [5]. In fact, this expression is related to Eq. (26) in an analogous way as was discussed previously for $D^{x x}$ and $D^{y y}$ on the one-hand side and $D_{i j}^{x}$ and $D_{i j}^{y}$ on the other side [16].

Although the considered B20 systems have cubic Bravais lattice, the elements $D^{x x}, D^{y y}$ and $D^{z z}$ are not the same because 


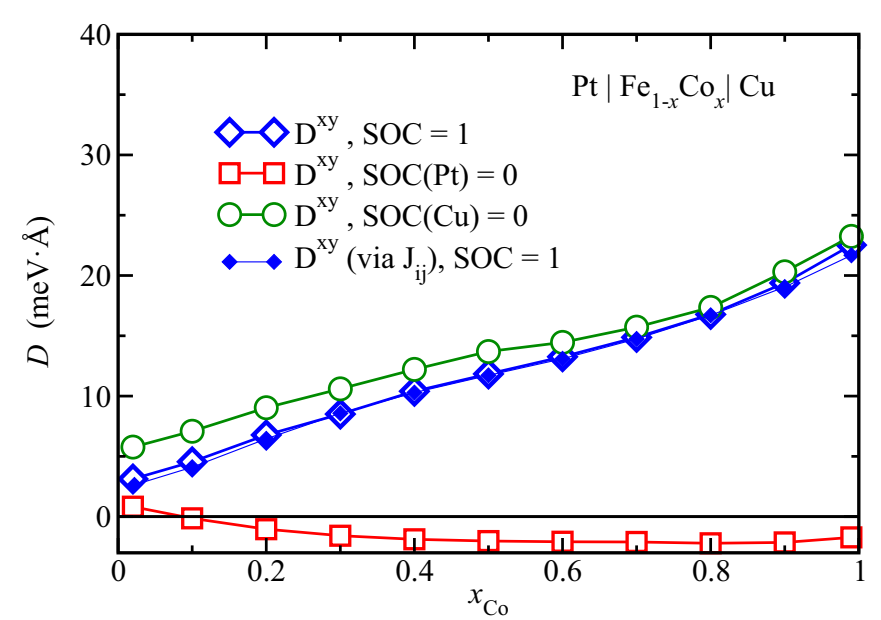

FIG. 3. The elements $D^{x y}$ of the micromagnetic DMI tensor for multilayer $\left(\mathrm{Cu} / \mathrm{Fe}_{1-x} \mathrm{Co}_{x} / \mathrm{Pt}\right)_{n}$ system as function of $\mathrm{Co}$ concentration.

of the reduced symmetry [23]. Nevertheless, the difference between all three components is rather small. This is a rather important result for the systems under consideration since the expression used for the calculation of the term $D^{z z}$ is rather different from the one used for the two other terms. It should be noted that the nonvanishing off-diagonal elements $D^{\mu \alpha}$ of the DMI tensor are substantially smaller than the diagonal elements [23] for the considered systems.

In contrast to the discussed B20 alloys, the symmetry of the $\left(\mathrm{Cu} / \mathrm{Fe}_{1-x} \mathrm{Co}_{x} / \mathrm{Pt}\right)_{n}$ multilayer system results in a vanishing of the diagonal elements of the micromagnetic DMI tensor and only the elements $D^{x y}$ and $D^{y x}$ are nonzero for the magnetization direction along $\hat{z}$, having opposite sign, $D^{y x}=-D^{x y}$. This is in line with the symmetry properties of the Fermi-sea contribution to the spin-orbit torque (SOT) tensor discussed previously by Wimmer et al. [24], that should be obeyed also by the DMI tensor due to the relationship between these two tensors $[11,23]$. The element $D^{x y}$ of the micromagnetic DMI tensor is plotted in Fig. 3 (open diamonds) as a function of Co concentration, exhibiting a monotonous increase with the increase of Co concentration. Almost the same behavior is shown by the results obtained via Eq. (32) (full diamonds) with a small deviation caused by the cutoff in the summation over the neighboring shells in this case.

Additional calculations have been performed to find out which atom type with its intrinsic SOC play the major role concerning the strength of the DMI in the multicomponent systems under consideration. In the case of B20 alloys the DMI strength is mainly determined by the SOC of the $3 d$ atoms. This follows immediately from a gradual decrease of the DMI when the SOC of these atoms is scaled to be zero. The $p$ states of Ge in the B20 materials are strongly hybridized with the $d$ states of the $3 d$ atoms and therefore have a key role in mediating the antisymmetric exchange interactions, as was previously discussed in the literature (see, e.g., Ref. [25]). In particular, the dependence of the DMI on the relative position of the $p$ states of $\mathrm{Ge}$ and $d$ states of the transition metals with respect to each other as well as with respect to the
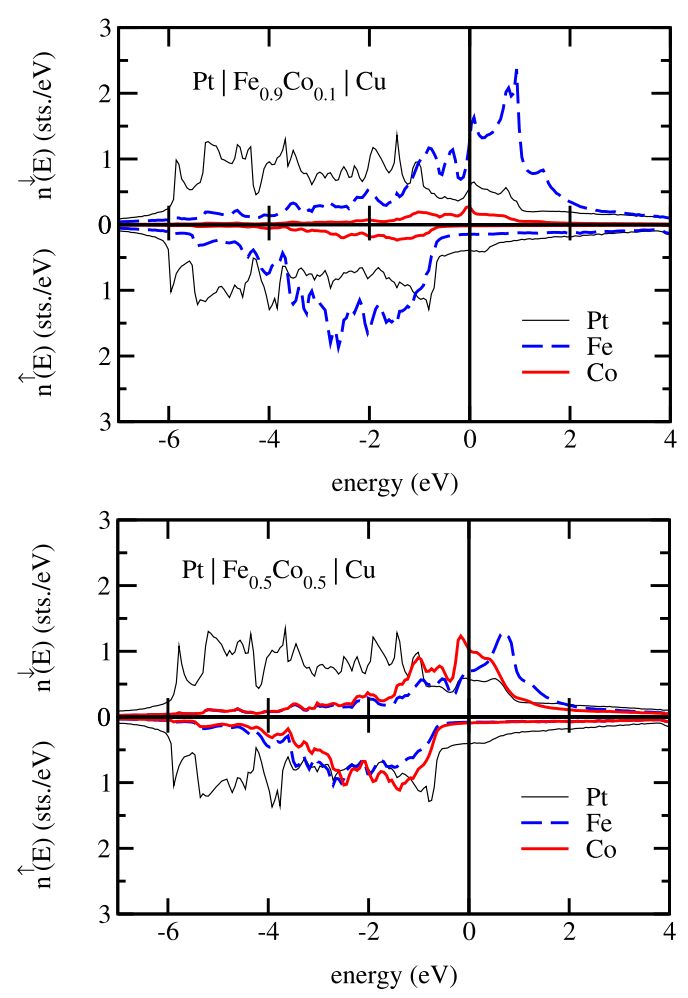

(a)
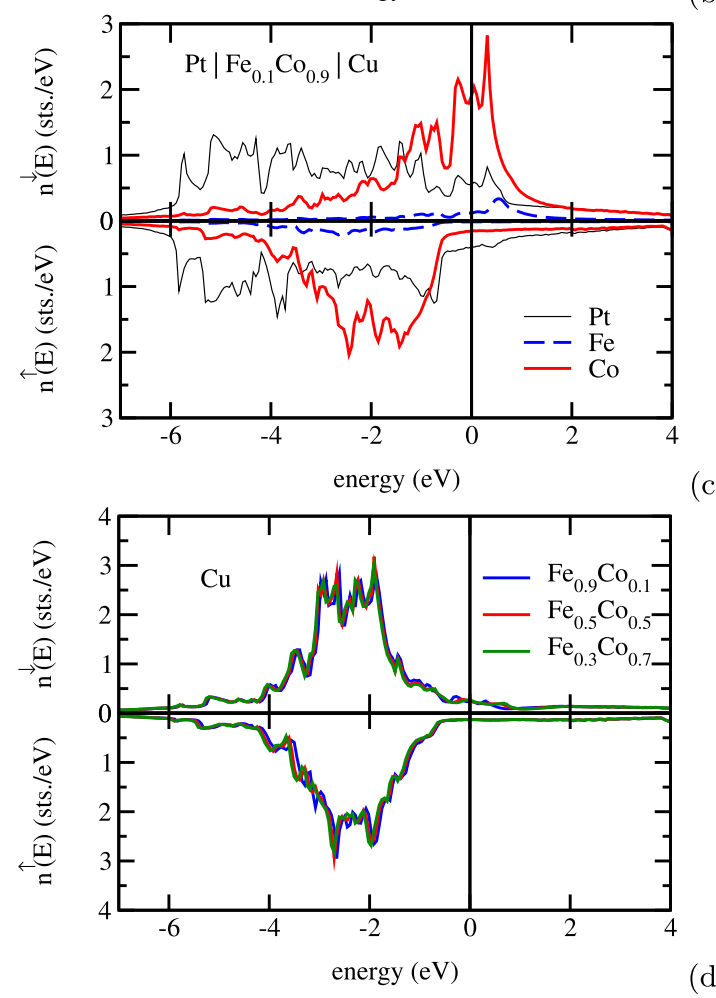

(c)

FIG. 4. Spin- and element-resolved $d$-DOS for the $d$ states in the multilayer system $\left(\mathrm{Cu} / \mathrm{Fe}_{1-x} \mathrm{Co}_{x} / \mathrm{Pt}\right)_{n}$ for $x=0.1$ (a), $x=0.5$ (b) and $x=0.9$ (c): Pt (black line), Co (red line), and Fe (blue line). The $d$-DOS for the $\mathrm{Cu}$ atoms in the systems are shown in (d) for three different concentrations.

Fermi energy, leading to a sign change of the DMI parameters upon variation of the composition in these alloys, has been demonstrated [25]. 
In the case of the multilayer system $\left(\mathrm{Cu} / \mathrm{Fe}_{1-x} \mathrm{Co}_{x} / \mathrm{Pt}\right)_{n}$, switching off the SOC for the $3 d$ atoms does not result in a significant change of the DMI. A similar result is found when the SOC for the $\mathrm{Cu}$ atoms is switched off (open circles); i.e., a weak increase of the DMI is seen almost over all the concentration region. However, the magnitude of the components $D^{x y}$ and $D^{y x}$ drops down when the SOC of the Pt atoms (open squares) is switched off and $D^{x y}$ even changes sign at $x \approx 0.1$. Obviously, one reason leading to this behavior is the strong SOC for the Pt atoms.

To allow for a more detailed discussion, we present in Fig. 4 the element resolved DOS of $\left(\mathrm{Cu} / \mathrm{Fe}_{1-x} \mathrm{Co}_{x} / \mathrm{Pt}\right)_{n}$. Figure 4(d) shows that the $\mathrm{Cu} d$ bands lie well below the Fermi energy. The fact that they are rather narrow indicates their relatively weak hybridization with the Fe and Co states. As a consequence this results in a weak modification of the $d$ states of $\mathrm{Cu}$, when the magnetic moments of the $3 d$ atoms within the $(\mathrm{Fe}, \mathrm{Co})$ layer are rotated to form a spin spiral in the system. In addition, one can say that the SOC, which is responsible for the antisymmetric exchange, is rather small for the $3 d$ states of the $\mathrm{Cu}$ atoms. Thus, both properties lead to a small contribution of the $\mathrm{Cu}$ layer to the DMI strength. On the other side, the heavy $\mathrm{Pt}$ atoms are characterized by a strong SOC. The partially occupied Pt $d$ bands are rather broad and substantially overlap in energy with the Co and Fe energy bands. This results (see Fig. 4) in a strong spin-dependent hybridization of the $\mathrm{Pt}$ states with the states of Co (more pronounced) and $\mathrm{Fe}$ (less pronounced) that in turn leads to a stronger modification of the Pt states due to a tilting of the spin moments in the $(\mathrm{Co}, \mathrm{Fe})$ layer, in particular, upon creation of a spin spiral. The lack of inversion symmetry results in different changes of the SOC-induced anticrossing gaps associated with spin spirals having different spin helicity, which at the end determines the sign and magnitude of the DMI vector (see Ref. [26]). A larger exchange splitting and, as a result, larger spin moment of the Co atoms can be responsible for a stronger DMI in the Co-rich limit due to a stronger perturbation caused in the neighboring layers upon rotation of the Co magnetic moments.

\section{SUMMARY}

In summary, we present in this work a general approach for the calculation of the spin-wave stiffness and DMI parameters based on perturbation theory expressed in terms of Green functions. Considering spin waves with small $\vec{q}$-vectors as a perturbation, the total energy expansion in powers of $q$ gives access to corresponding expressions for these quantities which have been worked out within the framework of the fully relativistic KKR Green function method. The expression for the spin-wave stiffness obtained this way can be seen as a relativistic extension of the nonrelativistic expression reported previously in the literature [1]. In particular, it is shown that it is given by the second-order term of the energy expansion. This holds also for the DMI elements $D^{z \alpha}$ which are not delivered in a first-order approximation and appear only via the second order energy term. In contrast, the DMI elements $D^{x \alpha}$ and $D^{y \alpha}$ are associated with the first-order term of the energy expansion. As a consequence, the expressions for the $D^{x \alpha}$ and $D^{y \alpha}$ elements differ from that for $D^{z \alpha}$. Despite this difference, we demonstrate that these expressions give almost the same results in the cases when this is required by the symmetry of the system. We also demonstrate full agreement between the results obtained using explicit expression for the spin stiffness and DMI tensors with those based on the interatomic exchange and DMI parameters.

\section{ACKNOWLEDGMENTS}

Financial support by the DFG via SFB 1277 (Emergent Relativistic Effects in Condensed Matter-From Fundamental Aspects to Electronic Functionality), as well as the DFG financial support via the priority programs EB154/36-1 are gratefully acknowledged.
[1] A. I. Liechtenstein, M. I. Katsnelson, V. P. Antropov, and V. A. Gubanov, J. Magn. Magn. Mater. 67, 65 (1987).

[2] V. Antropov, M. Katsnelson, and A. Liechtenstein, Physica B: Condens. Matter 237, 336 (1997).

[3] J. Kübler, Theory of Itinerant Electron Magnetism, International Series of Monographs on Physics (Oxford University Press, Oxford, 2009).

[4] L. Udvardi, L. Szunyogh, K. Palotás, and P. Weinberger, Phys. Rev. B 68, 104436 (2003).

[5] H. Ebert and S. Mankovsky, Phys. Rev. B 79, 045209 (2009).

[6] R. Zeller, J. Phys.: Condens. Matter 20, 294215 (2008).

[7] A. Bogdanov and A. Hubert, J. Magn. Magn. Mater. 138, 255 (1994).

[8] J. Kübler, Theory of Itinerant Electron Magnetism (Oxford University Press, Oxford, 2000), p. 460.
[9] J. Hamrle, O. Gaier, S.-G. Min, B. Hillebrands, Y. Sakuraba, and Y. Ando, J. Phys. D: Appl. Phys. 42, 084005 (2009).

[10] A. I. Liechtenstein, M. I. Katsnelson, and V. A. Gubanov, J. Phys. F: Met. Phys. 14, L125 (1984).

[11] F. Freimuth, S. Blügel, and Y. Mokrousov, J. Phys.: Condens. Matter 26, 104202 (2014).

[12] A. H. MacDonald and S. H. Vosko, J. Phys. C: Solid State Phys. 12, 2977 (1979).

[13] M. E. Rose, Relativistic Electron Theory (Wiley, New York, 1961).

[14] H. Ebert, J. Braun, D. Ködderitzsch, and S. Mankovsky, Phys. Rev. B 93, 075145 (2016).

[15] S. Mankovsky, S. Wimmer, and H. Ebert, Phys. Rev. B 98, 104406 (2018).

[16] S. Mankovsky and H. Ebert, Phys. Rev. B 96, 104416 (2017). 
[17] T. Koretsune, N. Nagaosa, and R. Arita, Sci. Rep. 5, 13302 (2015).

[18] S. Mankovsky, S. Bornemann, J. Minár, S. Polesya, H. Ebert, J. B. Staunton, and A. I. Lichtenstein, Phys. Rev. B 80, 014422 (2009).

[19] I. Nakai, J. Phys. Soc. Japan 52, 1781 (1983).

[20] M. Hatherly, K. Hirakawa, R. D. Lowde, J. F. Mallett, M. W. Stringfellow, and B. H. Torrie, Proc. Phys. Soc. (London) 84, 55 (1964).

[21] G. I. Rusov, Sov. Phys. Solid State 9, 146 (1967).
[22] S. Mankovsky, G. H. Fecher, and H. Ebert, Phys. Rev. B 83, 144401 (2011).

[23] S. Mankovsky, S. Wimmer, S. Polesya, and H. Ebert, Phys. Rev. B 97, 024403 (2018).

[24] S. Wimmer, K. Chadova, M. Seemann, D. Ködderitzsch, and H. Ebert, Phys. Rev. B 94, 054415 (2016).

[25] J. Gayles, F. Freimuth, T. Schena, G. Lani, P. Mavropoulos, R. A. Duine, S. Blügel, J. Sinova, and Y. Mokrousov, Phys. Rev. Lett. 115, 036602 (2015).

[26] L. M. Sandratskii, Phys. Rev. B 96, 024450 (2017). 